

\section{E-2688}

SOME EFFECTS OF BIAS ERRORS IN

REDUNDANT FLIGHT CONTROL SYSTEMS

by

Robert F. Stengel

June 1972

CHARLES STARK DRAPER LABORATORY

MASSACHUSETTS INSTITUTE OF TECHNOLOGY

CAMBRIDGE, MASSACHUSETTS

Approved: Daakd C Feaser 28 Jane 1972 D. C. FRASER, DIRECTOR, CONTROL AND FLIGHT DYNAMICS APOLLO GUIDANCE AND NAVIGATION PROGRAM

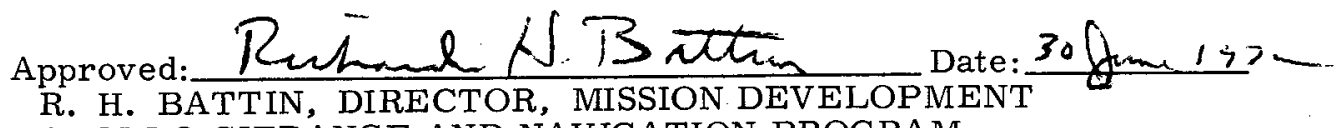
APOLLO GUIDANCE AND NAVIGATION PROGRAM

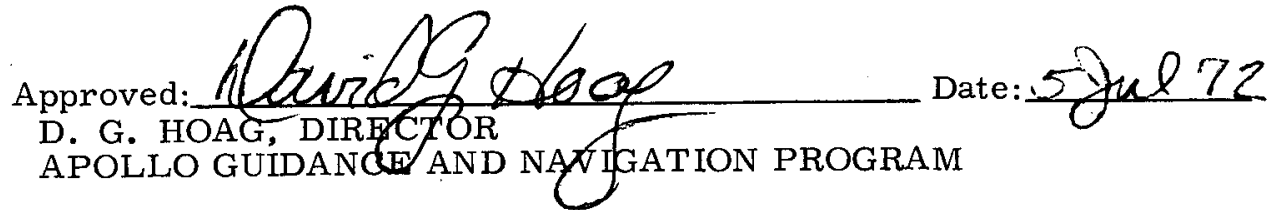

approved: Ralgh Q Aagan R. R. RAGAN,CDEPUTY DIRE ETOR Date: 5 fol 72 CHARLES STARK DRAPER LABORATORY 


\title{
SOME EFFECTS OF BIAS ERRORS IN REDUNDANT FLIGHT CONTROL SYSTEMS*
}

\author{
Robert F. Stengel ${ }^{* * *}$ \\ Charles Stark Draper Laboratory \\ Massachusetts Institute of Technology \\ Cambridge, Massachusetts
}

\section{Abstract}

The controllability and steady-state response of parallel-redundant flight control systems are examined. It is found that state components which appear in the parallel signal paths, e.g., individual actuator commands, are not controllable, although the sum of the command signals is well-behaved. If the response modes associated with these components are not stable, bias errors can cause the components to diverge, leading to the possibility of "nuisance trips" in failure detection/isolation logic and eventual control system lockup (at saturation). Combining the inputs to the control computers assures that sensor bias will not cause divergence, while cross-strapping control strings bound s divergent response to all bias error inputs. Results of numerical solutions confirm the problem and its solutions.

\section{Introduction}

In order to achieve acceptable levels of reliability and to meet fail-operational requirements, electronic ("fly-by-wire") and automatic-landing control systems require redundant strings of control system elements (a control string is a single replication of the sensors, logic and effectors necessary for vehicle control). During time-critical phases of a flight, e.g., a final approach in conditions of poor visibility, or for aircraft which depend on the control system for safety-of-flight (controlconfigured vehicles), each control string must continuously provide current actuator command solutions. These command solutions form a basis for failure detection (through comparison or voting) which is independent of the control law and which does not rely on built-in test procedures. Under certain conditions, however, the simultaneous solutions from individual strings can diverge, even though every string is operating without failure. In such an instance, the failure-detection logic may select out one or more strings, causing a so-called "nuisance trip". Nuisance trips lower the margin of safety and could cause unnecessaryaborts. They can occur in systems of control strings which are well-designed individually, leading to a requirement for channel equalization and/or restrictions on multiple-string operation. ${ }^{1,2}$

The principal reason that the control strings of a redundant system candiverge is that each string contains errors, however small, which are not the same as the errors in the other strings. At the same time, each string senses the same vehicle motion and (ultimately) commands the same control

* This research was supported by NASA Contract NAS9-10268.

** Group Leader - Member, AIAA. effector. A properlydesigned string operating alone would control the vehicle and limit its own errors; however, when confronted with the errors of many strings and a single effector to control them, the redundant system can become uncontrollable in the rigorous sense, as will be shown in the next section. The net command to the control effector, whether obtained by averaging, mid-value selection, or active-standby selection, may be well-behaved while one or more individual commands diverge. This behavior is illustrated by the results of numerical simulation in a later section.

If measurements are not perfect and if operating time is not so short that the limits of divergence are acceptable, the redundant control system must be protected against nuisance trips. The following sections of this paper will show that there are three ways of protecting against actuator command divergence caused by system errors. The most restrictive solution is to assure that the redundant control elements do not contain pure integration, i.e., that they do not introduce new components to the system state. The second solution is to feed identical control error signals to each set of control computers. The third method is to stabilize the uncontrollable modes of the control loops by "equalization" or "cross-strapping".

\section{Controllability of Redundant Systems}

The purpose of this section is to show that parallel-redundant control systems are not completely controllable if any of the system's state components appear in the redundant paths. This result is completely general, in that no amount of coupling or feedback restores complete controllability to the system (although such coupling can indeed have a beneficial effect).

A dynamical system is said to be completely controllable if the $n$ components of its state, $x(t)$, can be driven from an arbitrary initial condition, $x(0)$, to null in finite time, $T$, by the application of $\bar{a}$ suitable control history, $u(t), 0 \leq t<T$, where $\underline{u}(t)$ is the p-vector of control inputs to the dynamical system. For the linear, time-invariant system described by

$$
\underline{\dot{x}}(t)=\underline{F} \underline{x}(t)+\underline{\underline{G}} \underline{\underline{u}}(t)
$$

a necessary and sufficient condition for complete controllability is that the matrix, $\Gamma$, defined by

$$
\stackrel{r}{=}=\left[\underline{G}, \underline{F} \underline{\underline{G}}, \ldots \ldots \ldots, \underline{F}^{(n-1)} \underline{\underline{G}}\right]
$$

be of rank $n$, i.e., that it contains at least one non-zero $n \times n$ determinant. ${ }^{3}$ In such a case, $u(t)$ is said to span the state space, for its influence can be felt by all of the state components. 
A prototype for dual-redundant control is illustrated in Fig. 1. Each sensor and controller

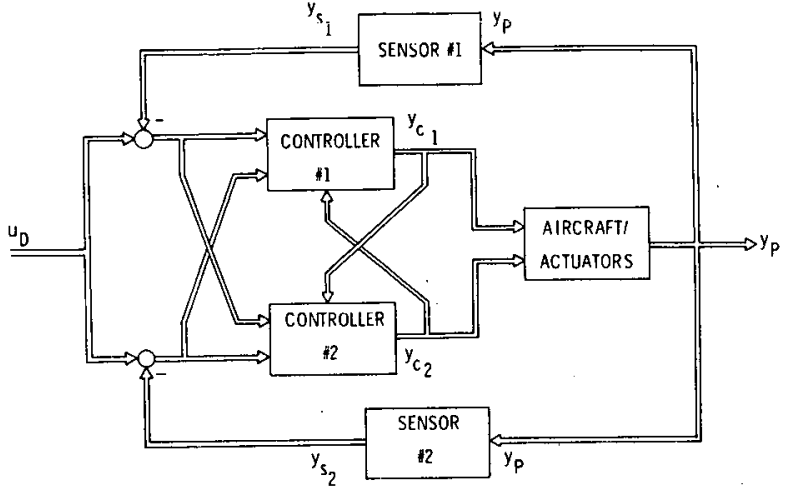

Fig. 1. State-space diagram of a fully-coupled, redundant control system.

of the corresponding $m-$ redundant system, as well as the single aircraft/actuator system, is represented by a matrix equation for the states, $x$, with constant-coefficient matrices:

$$
\begin{aligned}
& \dot{\dot{x}}_{S_{i}}=\underline{F}_{S}{\stackrel{x}{x_{i}}}_{i}+\underline{G}_{S} \underline{u}_{S_{i}}, i=1, m \\
& \dot{\underline{x}}_{C_{i}}=\underline{F}_{C} \underline{x}_{C_{i}}+\underline{G}_{C}{\stackrel{u}{C_{i}}}_{i}, i=1, m \\
& \dot{x}_{A}={ }_{\underline{F}} \underline{x}_{A}+\underline{G}_{A} \underline{u}_{A}
\end{aligned}
$$

These can be observed through the corresponding measurement vectors, $y$ :

$$
\begin{aligned}
& \underline{y}_{S_{i}}=\stackrel{M}{=} \underline{x}_{S_{i}}+\stackrel{N}{N}_{S} \stackrel{u}{S}_{i}, i=1, m \\
& \underline{y}_{C_{i}}=\stackrel{M}{=} C \underline{x}_{C_{i}}+{ }_{N}^{N} C \underline{u}_{C}, i=1, m \\
& \underline{y}_{A_{i}}=\stackrel{M}{=} \stackrel{x}{A}_{A}
\end{aligned}
$$

Referring to Fig. 1, it can be seen that the inputs, $u$, to each component of this feedback system are comprised of the outputs of the other components plus the desired input, $u_{D}$. As the system is posed here, it allows for multiple-inputs and multiple-outputs as well as higher-order dynamics in any element. It further specifies that each redundant element is identical to the other elements of like kind and that the outputs of the control strings are summed at the actuator. The aircraft/actuator measurement vector (Eq. 8) does not contain the in puts to that block; however, Fig. 1 shows that these are available for cross-feed to other control strings, and they are implicitly available for feedback within the controller through the definition of $\mathrm{F}_{\mathrm{C}}$. Using this figure and letting $m=2$, the dynamical equations (Eq. 3 - 5) become

$$
\begin{aligned}
& \left.\dot{\underline{x}}_{\mathrm{S}_{1}}=\underline{\mathrm{F}}_{\mathrm{S}} \underline{\mathrm{x}}_{\mathrm{S}_{1}}+\underline{\mathrm{G}}_{\mathrm{S}} \stackrel{(\mathrm{M}}{=} \underline{\mathrm{x}}_{\mathrm{A}}\right) \\
& \left.\dot{\underline{x}}_{\mathrm{S}_{2}}=\underline{\mathrm{F}}_{\mathrm{S}} \underline{\mathrm{x}}_{\mathrm{S}_{2}}+\underline{\mathrm{G}}_{\mathrm{S}} \stackrel{(\mathrm{M}}{=} \underline{\mathrm{x}}_{\mathrm{A}}\right) \\
& \underline{\dot{x}}_{C_{1}}=\underline{F}_{C} \underline{x}_{C_{1}}+\underline{\underline{G}}_{C}\left[2\left(\underline{u}_{D}-\underline{N}_{S} \underline{M}_{A} \underline{x}_{A}\right)-\underline{M}_{S}\left(\underline{x}_{S_{1}}+\underline{x}_{S_{2}}\right)\right]
\end{aligned}
$$

$$
\begin{aligned}
& \dot{\underline{x}}_{C_{2}}=\underline{F}_{C} \underline{x}_{C_{2}}+\underline{G}_{C}\left[2\left(\underline{u}_{D}-\stackrel{N}{N}_{S} \stackrel{M}{M}_{A} \underline{x}_{A}\right)-\underline{M}_{S}\left(\underline{x}_{S_{1}}+\underline{x}_{S_{2}}\right)\right]
\end{aligned}
$$

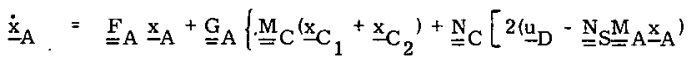

$$
\begin{aligned}
& \left.\left.\left.-\underline{M}_{\mathrm{S}} \underline{\mathrm{x}}_{\mathrm{S}_{1}}+\underline{\mathrm{x}}_{\mathrm{S}_{2}}\right)\right]\right\}
\end{aligned}
$$

These equations can be combined in a single matrix equation for a state vector which consists of all plant and controller components. As an intermediate step, the state components of each control string can be combined in a single vector, and the dynamical equation can be expressed in the following partitioned form:

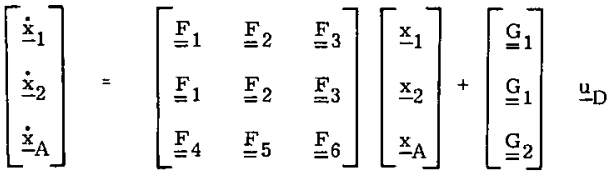

The order of the combined system is $n=m q+r$, where $m$ is the redundancy level, $q$ is the number of sensor-plus-controller state components and $r$ is the number of aircraft and actuator state components.

From Eq. (9 - 12), the first two rows of matrix coefficients in Eq. (14) are identical; hence, the observability matrix, $\underline{\underline{I}}$, of Eq. (2) takes the form,

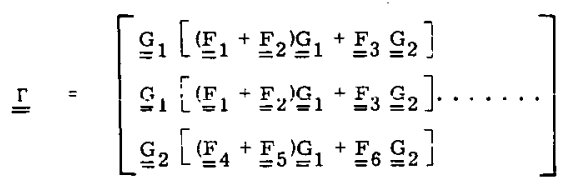

It can be seen that the top two rows of matrix coefficients in $\Gamma$ are identical and that, for $q$-order control strings, at least q rows will be duplicated in the dual-redundant version. Duplication of even one row in $\Gamma$ reduces its rank below $n$; therefore, if any of the system states occur in the identical control strings, the most general redundant system cannot be completely controllable.

A simple example illustrates the point. Let the aircraft/actuator dynamics consist only of a first-order lag, while the sensor has no dynamic effect and the controller consists of proportionalplus-integral compensation. The non-redundant system of equations is

$$
\left[\begin{array}{c}
\dot{x}_{C} \\
\dot{x}_{A}
\end{array}\right]=\left[\begin{array}{ll}
0 & -g_{C} \\
g_{A} & \left(f_{A}-g_{A}\right)
\end{array}\right]\left[\begin{array}{l}
x_{C} \\
x_{A}
\end{array}\right]+\left[\begin{array}{l}
g_{C} \\
g_{A}
\end{array}\right] u_{D}
$$

and it has the controllability matrix

$$
\sqsubseteq=\left[\begin{array}{cc}
g_{C} & -g_{C} g_{A} \\
g_{A} & {\left[\left(f_{A}-g_{A}\right) g_{A}-g_{C} g_{A}\right]}
\end{array}\right]
$$

The determinant of Eq. (17) is, in general, not equal to zero, and the system is completely controllable. Adding a second control string (without cross-strapping) gives an equation of the form 


$$
\left[\begin{array}{c}
\dot{x}_{C_{1}} \\
\dot{x}_{C_{2}} \\
\dot{x}_{A}
\end{array}\right]=\left[\begin{array}{ccc}
0 & 0 & -c \\
0 & 0 & -c \\
b & b & a
\end{array}\right]\left[\begin{array}{c}
x_{C_{1}} \\
x_{C_{2}} \\
x_{A}
\end{array}\right]+\left[\begin{array}{c}
c \\
c \\
2 b
\end{array}\right] u_{D}
$$

The determinant of the corresponding $\Gamma$ is of the form

$$
\stackrel{i \mathrm{i}}{=}|=\quad \underline{\underline{G}}, \underline{F G}, \underline{\underline{F}} \underline{\underline{F}} \underline{\underline{G}}|=\left|\begin{array}{lll}
\mathrm{C} & \mathrm{e} & \mathrm{g} \\
\mathrm{C} & \mathrm{e} & \mathrm{g} \\
2 \mathrm{~b} & \mathrm{~d} & \mathrm{f}
\end{array}\right|=0
$$

and the dual-redundant version is not completely controllable. Cross-strapping would eliminate the zeros in Eq. (18) but would not change the form of $\underline{\underline{\Gamma}}$.

It might be pointed out that a number of parallel-redundant autoland systems are in daily use and that this is in seeming contradiction with the present result. One can readily deduce from these results that the uncontrollability is only partial and that it is connected with the controller states. The airplane/actuator state components are as controllable as they were for simplex control, but intermediate components, e.g., actuator commands and controller error signals, may not be.

Reference 3 points out that a partially controllable system can be acceptable if the uncontrollable part is stable. Alternatively, an unstable or neutrally stable system could be acceptable with the assurance that the offending modes would never be excited. Looking again at the example of Eq. (18), the characteristic equation of the dual-redundant system without cross-strapping is

$$
|s \underline{\underline{I}}-\underline{\underline{F}}|=\left|\begin{array}{ccc}
s & 0 & c \\
0 & s & c \\
-b & -b & (s-a)
\end{array}\right|=s\left(s^{2}-a s+2 b c\right)=0
$$

The neutral root represented by the "free" s in $\mathrm{Eq} .(20)$ is a potential source of trouble, since it indicates a pure-integration response mode. Thus, a bias input can cause a ramp output. If, however, the outputs of the controllers are averaged, the difference between the actuator commands of the individual strings and the average can be used as an error signal which tells each string how far from the average its output lies. These error signals,

$$
\epsilon_{1}=x_{C_{1}}-\left(x_{C_{1}}+x_{C_{2}}\right) / 2 ; \epsilon_{2}=x_{C_{2}}-\left(x_{C_{1}}+x_{C_{2}}\right) / 2 \quad(21,22
$$

can be used to "equalize" the actuator commands if each is fed back to its controller with appropriate sign. The error signals are frequently integrated, for good long-term tracking of the redundant actuator commands, as in the SPZ-1 autoland system of the B-747.2 In the example of Eq. (18), a similar result is achieved by feeding the signals back to the respective controllers with a gain of $2 k$, yielding the characteristic equation

$$
\left|\begin{array}{ccc}
(s-k) & k & c \\
k & (s-k) & c \\
-b & -b & (s-a)
\end{array}\right|=s^{3}-(2 k+a) s^{2}+2(a k+b c) s-4 b c k=0 \quad(23
$$

Equation (23) can be factored to (s-2k) - $\left(s^{2}-a s+2 b c\right)=0$, and the neutral mode is stabil- ized for $k<0$. Further examination shows that the transfer function $x_{A}(s) / u_{D}(s)$ is the same with or without cross-strapping.

This section has shown that redundant control system sare never completely controllable but that cross-strapping (or equalization)can stabilize the uncontrollable modes (which are still uncontrollable) and, therefore, limit divergence.

\section{Steady-State Response to Bias Errors and Noise}

Single-input, single-output transfer functions are used here to describe the effects of bias and noise injected at the sensor, controller, and actuator inputs of a parallel-redundant control system. As in the previous section, the system is assumed to be linear and time-invariant; consequently, the combination of signals implies summing, although many of the results are qualitatively similar for mid-value selection. Independent-string, combined-input, and cross-strapped redundant systems are discussed in turn.

It is convenient to replace the dual-redundant model of the previous section with the independentstring block diagram of Fig. 2. In this figure,

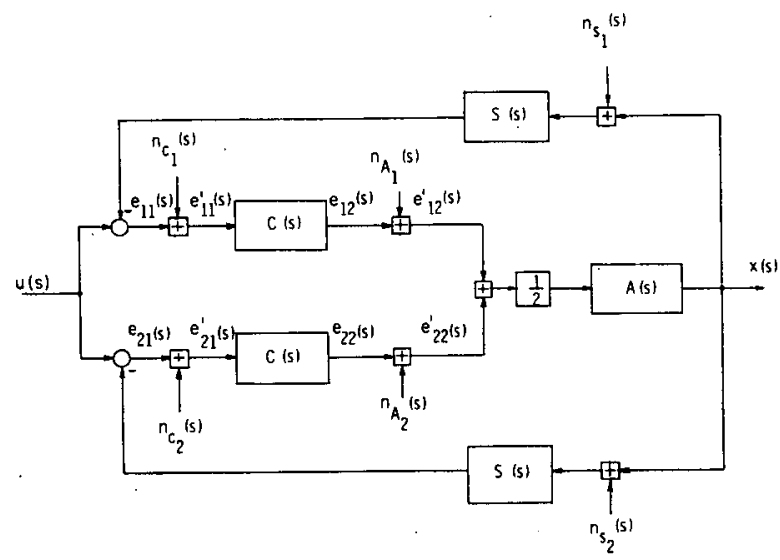

Fig. 2. Block diagram of an independent-string, dual-redundant flight control system

sensor, controller, and aircraft/actuator transfer functions are denoted by $\mathrm{S}(\mathrm{s}), \mathrm{C}(\mathrm{s})$, and $\mathrm{A}(\mathrm{s})$ respectively. The Laplace transforms of the system input and output are $u(s)$ and $x(s)$, while the $j^{\text {th }}$ control signal transform of the $i^{\text {th }}$ control string is $e_{i j}(s)$. The bias and noise inputs at the sensor, controller, and actuator for the $i^{\text {th }}$ system are $n_{S i}(s), n_{C i}(s)$, and $\mathrm{n}_{\mathrm{Ai}}(\mathrm{s})$. The actuator commands, $e_{i 2}(\mathrm{~s})$ are averaged at the actuator.

Using block-diagram algebra, the output transform of an m-redundant independent-string system is

$$
x=A\left\{\frac{1}{m} \sum_{i=1}^{m} n_{A_{i}}+C\left[u+\frac{1}{m} \sum_{i=1}^{m}\left(n_{C_{i}}-S n_{S}\right)\right]\right\} /(1+A C S)
$$

It can be seen that $x(s) / u(s)=A C /(1+A C S)$, as expected, and that each of the error inputs is attenuated by the averaging process.

The intermediate control signals, which consist of $n$ controller inputs and $m$ actuator commands, can be expressed as the matrix 
The solution to Eq. (28) is

$\underline{\underline{E}(s)} \quad=\left[\begin{array}{cc}e_{11^{(s)}} & e_{12}(s) \\ e_{21}(s) & e_{22}(s) \\ \vdots & \vdots \\ e_{m 1}(s) & e_{m 2}(s)\end{array}\right]$

The $\mathrm{i}^{\text {th }}$ controller input transform is

$e_{i 1}=u-\frac{A S}{m}\left[\sum_{k=1}^{m}\left({ }^{C} C_{k}+n_{A_{k}}\right)+{ }^{n} C_{i}+C \sum_{k=1}^{m} e_{k 1}\right]$

$(26$

while the $i^{\text {th }}$ actuator command transform is

$$
e_{i 2}=c\left\{u+n_{C}-\frac{A S}{m}\left[n_{C}+\sum_{k=1}^{m}\left(n_{A_{k}}+e_{k 2}\right)\right]\right\}
$$

Equations 26 and 27 can be combined in the matrix equation

$$
\underline{\underline{\mathrm{B}}} \underline{\underline{\mathrm{E}}}=\underline{\mathrm{R}} \text {, }
$$

where

$$
\begin{aligned}
& \stackrel{B}{=}=\left[\begin{array}{cccc}
(1+\beta) & \beta & \ldots & \beta \\
\beta & (1+\beta) & \ldots & \beta \\
\ldots & \ldots & \beta & \beta \\
\beta & \beta & \ldots & (1+\beta)
\end{array}\right] \\
& \underline{\mathrm{R}}=\left[\underline{\mathrm{r}}_{1} \underline{\mathrm{r}}_{2}\right] \\
& \underline{r}_{1}=\left[\begin{array}{c}
u-S\left(p+n_{S_{1}}\right) \\
\vdots \\
u-S\left(\rho+n_{S_{m}}\right)
\end{array}\right] \\
& \underline{r}_{2}=c\left[\begin{array}{cc}
u+n_{C_{1}}-s\left(s+n_{S_{1}}\right) \\
\vdots & \\
u+n_{C_{m}}-s\left(s+n_{S_{m}}\right)
\end{array}\right]
\end{aligned}
$$$$
(\mathrm{m} \times \mathrm{m})
$$

and

$$
\begin{aligned}
& B=A C S / m \\
& \rho=\frac{A}{m} \sum_{k=1}^{m}\left({ }^{m} n_{C_{k}}+n_{A_{k}}\right) \\
& S=\frac{A}{m} \sum_{k=1}^{m} n_{A_{k}}
\end{aligned}
$$

130

$$
\underline{\underline{E}}=\underline{\underline{B}}^{-1} \underline{\underline{R}}
$$

where

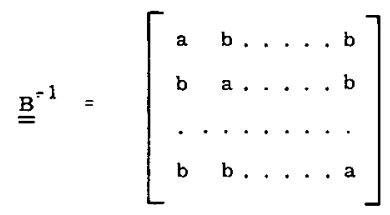

and

$$
a=[1+(m-1) \beta] /(1+m \beta)=\left[1+\left(\frac{m-1}{m}\right) A C S\right] /(1+A C S)
$$

$$
b=-\beta /(1+m \beta)
$$$$
=-\left(\frac{1}{\mathrm{~m}}\right) \mathrm{ACS} /(1+\mathrm{ACS})
$$

The control signal transforms of the first string are then

$$
\begin{aligned}
& e_{11}=a r_{11}+b r_{21}+\ldots+b r_{m 1}, \\
& e_{12}=a r_{12}+b r_{22}+\ldots+b r_{m 2} .
\end{aligned}
$$

and those of the remaining strings are similarly defined. The transfer functions between error inputs and control signals are obtained by substitution of the appropriate definitions in Eq. (40) and (41). -The transfer functions for the actuator command are particularly important. They can be expressed in terms of the basic closed-loop transfer function,

$$
H(s)=A C S /(1+A C S) \text {, }
$$

$(42$

which is assumed to be well-behaved and whose time response to a step input is bounded as time $\rightarrow \infty$. For a dual-redundant control system, the actuator command transfer functions are

$$
\begin{aligned}
& \frac{e_{12}(s)}{u(s)}=\frac{H}{A S} \\
& \frac{e_{12}(s)}{n_{A_{1}}(s)}=\frac{e_{12}(s)}{n_{A_{2}}(s)}=-.5 H
\end{aligned}
$$

$\frac{e_{12}(s)}{n_{S_{1}}(s)}=\frac{-(1+.5 A C S) H}{A}$

$$
\frac{e_{12}(s)}{n_{S_{2}}(s)}=.5 \mathrm{CSH}
$$

$$
\frac{e_{12}(s)}{n_{C_{1}}(s)}=\frac{(1+.5 A C S) H}{A S}
$$

$$
\frac{\mathrm{e}_{12}(\mathrm{~s})}{\mathrm{n}_{\mathrm{C}_{2}}(\mathrm{~s})}=-.5 \mathrm{CH}
$$


By the final-value theorem, the limit of actuator command response to a unit-step error input is

$$
\lim _{t \rightarrow \infty} e(t)=\lim _{s \rightarrow 0} s \frac{e(s)}{n(s)}\left(\frac{1}{s}\right)=\lim _{s \rightarrow 0} \frac{e(s)}{n(s)}
$$

If any transfer function contains an " $s$ " $\mathrm{di}-$ visor, the corresponding actuator command response is unbounded. This might occur in a variety of ways, but the most common would be integration in the sensor or controller. In the case of proportional-plus-integral compensation, $\mathrm{C}(\mathrm{s})=\mathrm{k}(\mathrm{s}+\alpha) / \mathrm{s}$, and Eq. (45) to (48) show that sensor or controller biases cause the actuator command to diverge. Response to sensor bias in the first string (Eq. (45)) causes negative divergence, while a bias in the second string (Eq. (46)) causes positive divergence. By similarity, a bias in the first string's sensor causes negative divergence in the first string and positive divergence in the second string; hence, the averaged output of the two actuator commands is well-behaved. The same response pattern obtains for controller bias. The steady-state rate of divergence is found by multiplying Eq. $(45-48)$ by $s$ and taking the limitas $\mathrm{s} \rightarrow 0$. Consequently, higherorder integral compensation causes a higher degree of divergence in the redundant system.

This result assumes that there is no communication between the control strings. If, however, the inputs from each string are averaged and sent to every string, there is a common controller input signal given by

$$
e_{1}(s)=\left\{u-\frac{S}{m} \sum_{k=1}^{m}\left[n_{S_{k}}+A\left(n_{A_{k}}+C n_{C_{k}}\right)\right]\right\} /(1+A C S)
$$

and the $i^{\text {th }}$ actuator command transform is

$$
e_{i 2}(s)=C\left\{u+n_{C_{i}}-\frac{s}{m} \sum_{k=1}^{m}\left[n_{S_{k}}+A\left(n_{A_{k}}+e_{k 2}\right)\right]\right\}
$$

The previous equations can be used, letting $\underline{\underline{\mathrm{R}}}=\underline{\mathrm{r}}_{2}$ and

$$
\underline{r}_{2}=C\left[\begin{array}{c}
u+n_{C_{1}}-\epsilon \\
u+n_{C_{m}}-\epsilon
\end{array}\right]
$$

with

$$
\epsilon=\frac{S}{m} \sum_{k=1}^{m}\left(n_{S_{k}}+A n_{A_{k}}\right)
$$

Combining the sensor outputs, as shown in Fig. 3(a), has no effect on the actuator command response to desired input, actuator error and controller error; however, the transfer functions for dual-redundant response to sensor error become

$$
\frac{e_{12}(s)}{n_{S_{1}}(s)}=\frac{e_{12}(s)}{n_{S_{2}}(s)}=-.5 \mathrm{H} / \mathrm{A} .
$$

In this case, the response to sensor bias is independent of the compensation, and the most important form of actuator command divergence can be avoided. Combination of controller inputs succeeds in eliminating the sensor bias-induced divergence because each string has the same response to a particular error. It has no effect on errors arising in the controllers, which are "downstream" of the combining process.

The third alternative, cross-strapping the actuator commands, allows each string to sense the commands of the other strings. Choosing the difference between the individual command and the average command as the feedback variable,

$$
e_{i 3}=e_{i 2}-\frac{1}{m} \sum_{k=1}^{m} e_{k 2}
$$

The controller output, as well as the actuator input, should be bounded; furthermore, the control compensation can have beneficial effect on the cross-strapping signal. It is assumed, therefore, that $e_{j}$ is fedback to its own system with compensation, $K(s)$, as illustrated by Fig. 3(b). The controller

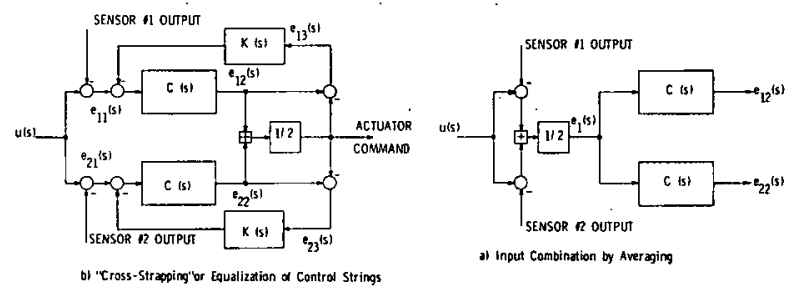

Fig. 3 Methods of coupling redundant control strings

input transform is unchanged by this modification, but the $i^{\text {th }}$ actuator command transform becomes

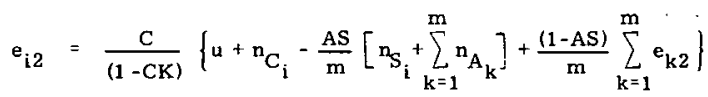

$\underline{B}$ and $\underline{r}_{2}$ (Eq. (28) and (32)) are modified by the cross-strapping, as indicated by Eq. (56); however, the most important effect of cross-strapping can be deduced from Eq. (56), noting that the multiplier of both $n_{C_{i}}$ and $n_{S_{i}} C /(1-C K)$ rather than C. As a consequence, the multiplier is bounded as $s \rightarrow 0$, and response to sensor and controller errors is no longer divergent.

\section{Results of Numerical Simulation}

A model for a triply-redundant digital flight control system for the space shuttle orbiter is used to illustrate the effects of a pitch-angle measurement bias during landing approach. The linear, time-invariant differential equations for longitudinal motion are integrated numerically to obtain the se results. In all cases, a $1^{\circ}$ pitch bias is imposed at $t=1$ sec. This large bias would reflect an abnormal condition rather than a nominal error within system specifications; however, the model is linear, and the response modes are independent of the bias magnitude. Reducing the bias magnitude by a factor of 100 reduces the response magnitudes by the same factor. 
Redundant configurations with a variety of controller input and output combination philosophies have been simulated. In all cases, the control compensation of the fifth-order dynamic model consists of pitch-rate damping with proportionalplus-integral. pitch-attitude compensation. The triplex-actuator commands drive the model through an averaged command, a mid-value selected command, or through a single-string command. The three controllers receive independent, average, weighted-average, or mid-value selected sensor outputs. A series of runs with independent inputs, averaged outputs, and varying cross-strapping gains also is presented.

Figure 4 illustrates the effect of a pitch bias in one string on the elevator, pitch, and pitch-rate response of the vehicle. The average output of the

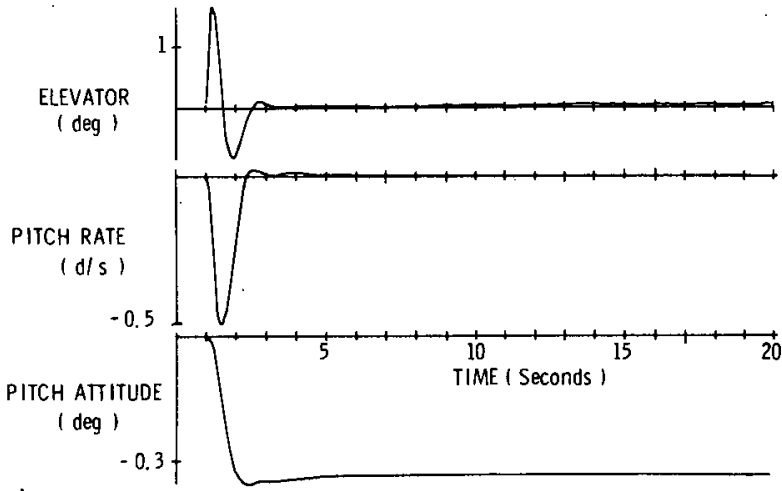

Fig. 4 The effect of a 1 pitch bias in one of three control strings. Elevator command is obtained by averaging the outputs of the redundant strings. This vehicle response is obtained with independent inputs, combined inputs, and cross-strapping.

three strings is well-behaved, and the pitch angle assumes a steady-state value which is $1 / 3$ the bias magnitude. This response pattern is the same for both independent- and averaged inputs to the three controllers. The actuator commands of the three strings with independent inputs are compared with the average command in Fig. 5. The negative divergence of the offending string is opposed by the remaining good strings, and the difference in commands would eventually cause a "nuisance trip" of the failure detection mechanism. Averaging the inputs as well as the outputs provides the response shown in Fig. 6, where all commands are seen to be well-behaved.

The problem of independent inputs without cross-strapping persists for active-standby configurations. If the biased string is controlling with the good strings in "standby", the good strings observe the vehicle's response to the measurement bias and generate opposing commands (which have no effect on the vehicle), as shown in Fig. 7. Figure 8 illustrates the converse, with a good string controlling and a biased backup string. In this case there is no vehicle response, but the backup com$m$ and diverges (reaching an imposed $-90^{\circ}$ limit on output signals).

These results are summarized and are compared with weighted-average and mid-value selection in Table $\mathrm{I}$, which lists command response at $t=20 \mathrm{sec}$. to a $1-\mathrm{deg}$ bias imposed on string \#1 at

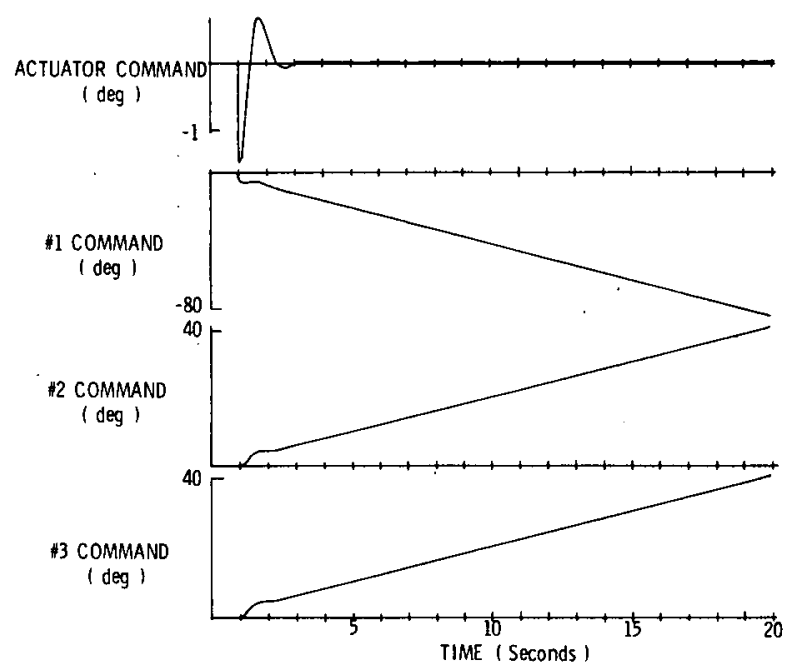

Fig. 5 Divergence of commands from individual strings in an independent-string triplex control configuration. (Sign of actuator command reversed by elevator servo model.)

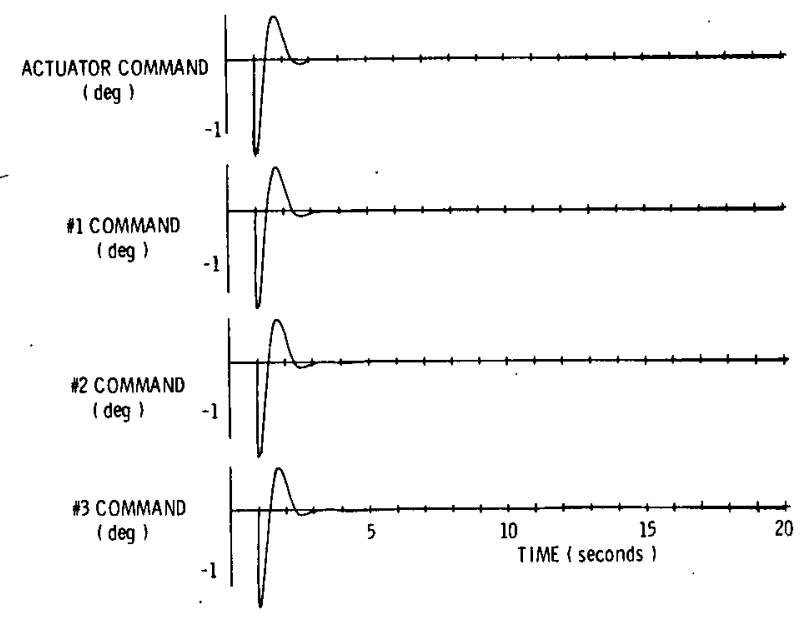

Fig. 6 Identical commands from triplex control strings with combined inputs.

$t=1$ sec. In no case does the combined actuator command diverge; how ever, all cases with independent inputs have individual string divergence. Both averaging and mid-value selection of the inputs prevent divergence. The latter has the additional advantage of rejecting the biased data, thus issuing no command in response to the single error.

A possible alternative to averaging which causes each string to rely on its own data most heavily is weighted averaging. In this approach, each controller uses $50 \%$ of its own sensor's output and $25 \%$ of each of the remaining outputs (rather than multiplying each by $1 / 3$ in the straight aver- 


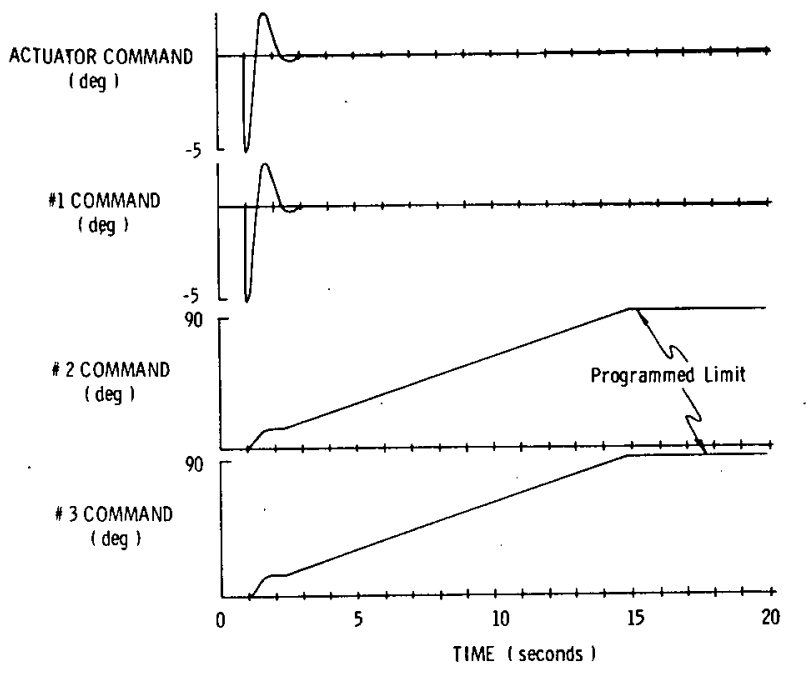

Fig. 7 Divergence of backup commands in triplex active-standby configuration when active string (\#1) has bias.

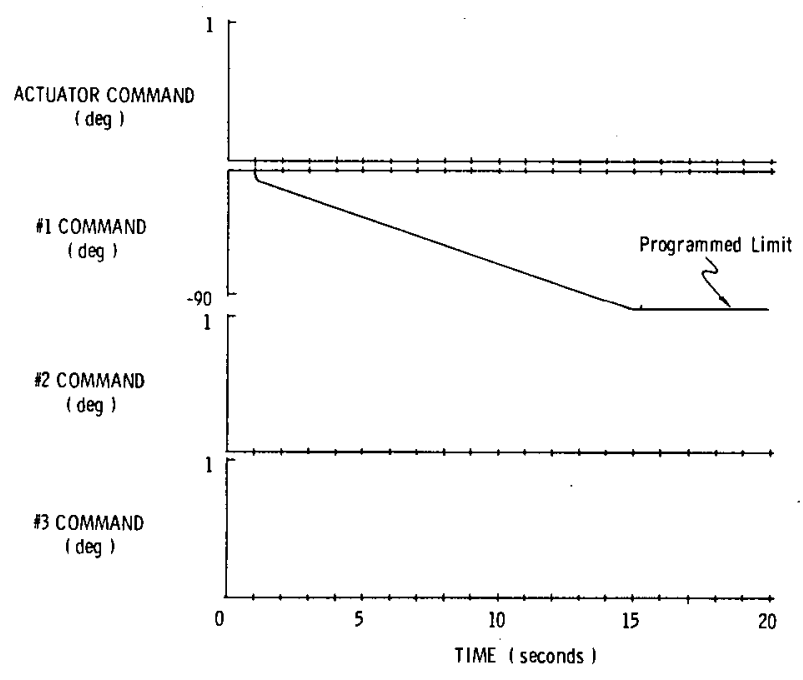

Fig. 8 Divergence of backup string with bias (\#1) in active-standby configuration (\#2 is active). Net command to system is zero.

aging approach). This scheme preserves a greater degree of autonomy for each string, giving better inherent protection against "hardover" failures (but it is not as good as mid-value selection in that respect). Unfortunately, weighted averaging does not eliminate bias-induced diver gence; it only slows it down. The reason is that weighted averaging does not prevent the neutrally stable mode introduced by integral compensation from being forced; this occurs only when all strings receive the same information.

Cross-strapping stabilizes the neutral mode, as shown by Fig. 9. It does not yield identical commands from each string, as input combination does, but it protects against errors within the controllers themselves. Table II indicates the level of commands from the three flight controllers at $\mathrm{t}=\mathbf{2 0}$. sec., and it provides a warning against using
Table I. Effect of Input and Output Combination on Actuator Command Bias Response (in deg.)

$\begin{array}{ccccc}\text { Input } & \text { Output } & \# 1 \text { Command } & \# 2, \# 3 \text { Command Actuator Command } \\ \text { Independent Average } & -80.7 & 40.3 & -0.046 \\ " & \text { Mid-Value } & -90 . & 0 . & 0 . \\ " & \text { String \# } 1 & -0.14 & 90 . & -0.14 \\ \text { ". } & \text { String \#2 } & -90 . & 0 . & 0 . \\ \text { Average } & \text { Average } & -0.046 & -0.046 & -0.046 \\ \text { " } & \text { Mid-Value } & -0.046 & -0.046 & -0.046 \\ \text { Mid-Value } & \text { Average } & 0 . & 0 . & 0 . \\ \text { " } & \text { Mid-Value } & 0 . & 0 . & 0 . \\ \text { Weighted } & \text { Average } & -20.2 & 10.0 & -0.048 \\ \text { Average } & & & & \end{array}$

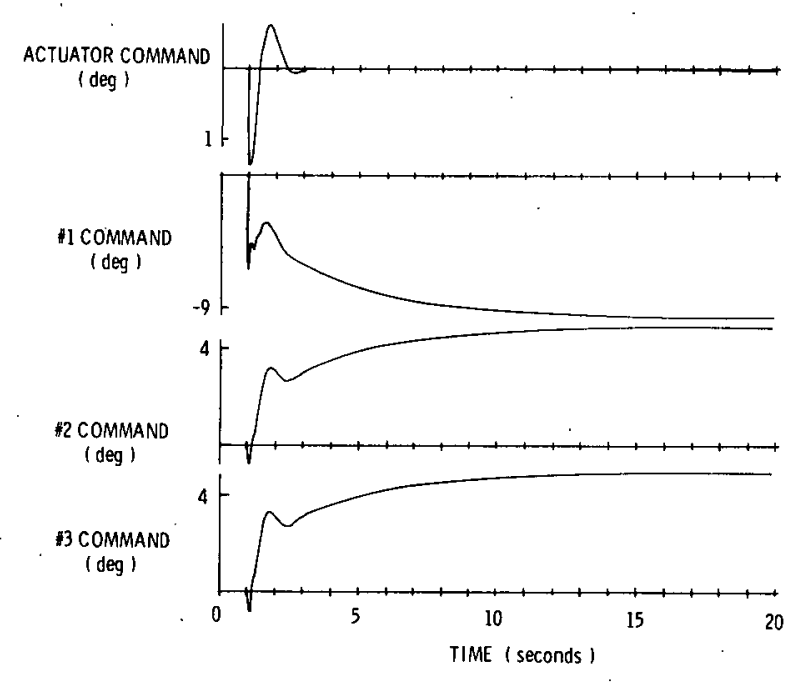

Fig. 9 Bounded response to pitch bias when control strings are coupled by equalization or cross strapping. (Coupling gain $=.05$. )

Table II. Effect of Cross-Strapping on Actuator Command Bias Response (in deg.)

Coupling Gain \#1 Command \#2,\#3 Command Actuator Command

$\begin{array}{llcc}0 . & -80.7 & 40.3 & -.046 \\ .01 & -36.4 & 18.1 & -.048 \\ .02 & -21 . & 10.7 & " \\ .05 & -9 . & 4.4 & " \\ .1 & -5.5^{*} & 2.7^{*} & "\end{array}$

\section{* Oscillatory}


too high a coupling gain with digital control. The cross-feed signal necessarily lags the controller compensation by one sampling period, causing oscillation of the individual commands at high gain (although the average does not exhibit the oscillation). The nearly steady-state difference of $13.4^{\circ}$ for a coupling gain of .05 is excessive, but then so is the $1^{\circ}$ bias. For more reasonable mea surement biases, cross-strapping should reduce the tracking error of the three strings to an acceptable level.

\section{Conclusion}

Good single-string control design does not assure that a redundant ver sion of the same system will be operationally acceptable. While it is relatively easy to obtain similar response to controls from both simplex and multiplex control systems, the individual behavior of the redundant control strings can fool failure detection logic, triggering "nuisance trips", and can lead to eventual control system lockup, when the individual strings have diverged to saturation. In those cases where reinitialization of actuator commands is feasible, it may be possible for redundant, independent control strings to provide an in-flight maintenance capability, but when safety-of-flight is dependent on correct control without interruption, further precautions must be taken. This is especially true for high-order control compensation.

It has been shown that redundant control systems can be completely controllable only if all system states are external to the redundant control paths, and that integral compensation, in particular, can cause command response to sensor or controller errors to be divergent. Combining sensor outputs so that each control computer receives the same inputs prevents divergence caused by sensor bias and allows actuator commands to track with zero relative error (assuming identical string dynamics). It does not protect against divergence due to controller bias. Cross-strapping, i.e., feeding the difference between individual commands and the average (or median) back to the respective controllers, bounds the errors due to both kinds of biases by stabilizing the uncontrollable modes of the redundant control loops. Proportional feedback does not eliminate command tracking error in the example shown here, although compensation of the crossfeeds could do so. It appears that both input combination and cross-strapping should be employed in the redundant control system to achieve design and operational flexibility.

\section{References}

1. Bishop, R. I., "Development of Airborne Hardware for Automatic Landing Systems," in Aircraft Landing Systems, NATO AGARD CP-59-70, Neuilly-sur-Seine, Sept., 1970, pp. $8-1$ to $8-12$.

2. Popik, M. J., "Automated Landing System," Sperry Rand Engineering Review, Vol. 24, No., 2, 1971, pp. 24-31.

3. DeRusso, P. M., Roy, R. J., Close, C. M., State Variables for Engineers, J. Wiley \& Sons, New York, 1965. 